\title{
Supplementing phytogenic compounds or autolyzed yeast modulates ruminal biogenic amines and plasma metabolome in dry cows experiencing subacute ruminal acidosis
}

\author{
E. Humer, ${ }^{*}$ I. Kröger, ${ }^{*}$ V. Neubauer, ${ }^{*} \dagger$ K. Schedle, $\ddagger$ N. Reisinger,§ and Q. Zebeli*1 \\ *Institute of Animal Nutrition and Functional Plant Compounds, and \\ †Institute for Milk Hygiene, Milk Technology and Food Science, Department for Farm Animals and Veterinary Public Health, \\ University of Veterinary Medicine, Veterinaerplatz 1, 1210 Vienna, Austria \\ †Institute of Animal Nutrition, Livestock Products, and Nutrition Physiology, Department of Agrobiotechnology (IFA-Tulln), \\ University of Natural Resources and Life Sciences, Vienna, Muthgasse 11/1, 1190 Vienna, Austria \\ $\S B I O M I N$ Research Center, BIOMIN Holding GmbH, Technopark 1, 3430 Tulln, Austria
}

\begin{abstract}
Subacute ruminal acidosis (SARA) causes ruminal dysbiosis, thereby increasing the risk of systemic metabolic disorders in cattle. We recently showed that supplementation with phytogenic compounds (PHY) or autolyzed yeast (AY) counteracted negative effects of SARA by improving ruminal $\mathrm{pH}$ and microbiome. This study investigated the effects of an intermittent SARA challenge on the ruminal concentration of biogenic amines (BA) and lipopolysaccharides (LPS), as well as on the blood metabolome. We also evaluated effects of PHY and AY on the latter variables. Eight rumen-cannulated nonlactating Holstein cows were arranged in an incomplete $4 \times 3$ Latin square design with 4 experimental runs and 3 treatment groups. During each run, cows were switched from an all-forage diet (baseline) to an intermittent concentrate-challenge diet with a forage:concentrate ratio of 35:65 (dry matter basis) to induce SARA for 1 (SARA1) or 2 (SARA2) $\mathrm{wk}$, separated by $1 \mathrm{wk}$ of forage-only feeding. The 3 treatment groups were no additive as control, PHY, or AY. During baseline, SARA1 and SARA2 rumen fluid samples were collected for analysis of BA and LPS. Blood samples were taken during baseline and SARA1 for a targeted metabolomics approach. Highconcentrate feeding caused a 9-fold increase in ruminal LPS during SARA1 and an 11-fold increase in SARA2 compared with the baseline. Elevated concentrations of ruminal BA were found during both SARA periods, with histamine showing the strongest increase during SARA1. Moreover, a decrease in phosphatidylcholines, lysophosphatidylcholines, sphingomyelines, and several AA in the blood during SARA1 were detected. Supple-
\end{abstract}

Received March 14, 2018.

Accepted May 31, 2018.

${ }^{1}$ Corresponding author: qendrim.zebeli@vetmeduni.ac.at mentation of PHY decreased concentrations of LPS $(-43 \%)$, histamine $(-66 \%)$, pyrrolidine $(-38 \%)$, and spermine $(-54 \%)$ in SARA1 and cadaverine in SARA2 $(-50 \%)$. Moreover, cows that received PHY had higher concentrations of cholesterol $(+26 \%)$, several AA, and phosphatidylcholines in SARA1 compared with control cows. For AY, decreases in ruminal ethanolamine $(-21 \%)$, methylamine $(-52 \%)$, histamine $(-54 \%)$, spermidine $(-44 \%)$, and spermine $(-80 \%)$ in SARA1 were observed, whereas in the blood an increase in tryptophan was noticed. In conclusion, the SARA was associated with markedly increased concentrations of LPS and BA in the rumen fluid and undesirable shifts in the plasma metabolome. Supplementation of PHY and AY counteracted some of these changes and therefore may help in attenuating negative effects of high-concentrate feeding in dairy cattle.

Key words: feed additive, subacute rumen acidosis, biogenic amine, blood metabolome, lipopolysaccharide

\section{INTRODUCTION}

Concentrate-rich diets have become a common practice in commercial cattle production increasing the risk of SARA. This digestive disorder is characterized by a prolonged and intermittent decline in ruminal $\mathrm{pH}$, causing major microbial imbalances in the rumen (Tajima et al., 2000; Khafipour et al., 2009), commonly known as dysbiosis. Growing evidence indicates that gastrointestinal dysbiosis during SARA promotes the production of microbe-derived toxic compounds, including bacterial LPS and biogenic amines (BA; Ametaj et al., 2010; Wang et al., 2013; Mao et al., 2016), which have been suggested as biomarkers of dysbiosis during SARA in cattle (Plaizier et al., 2012).

The release of large amounts of bacterial LPS and $\mathrm{BA}$ in the rumen has been reported during a single SARA bout (Ametaj et al., 2010; Saleem et al., 2012); 
however, it is not clear if the release of such toxic compounds differs with duration of SARA or when SARA occurs repeatedly. Recent research of our team has shown that intermittent SARA affects $\mathrm{pH}$ and causes important bacterial shifts in the rumen. These effects were stronger during the first versus the second SARA bout, despite feeding the same diet (Neubauer et al., 2018). Most interestingly, this research showed that supplementation of phytogenic compounds (PHY) or autolyzed yeast (AY) alleviated the dysbiosis mainly by reducing the drop of ruminal $\mathrm{pH}$, promoting chewing activity, and beneficially affecting microbial community structure. These effects were observed particularly when severest pH drops occurred (Kröger et al., 2017; Neubauer et al., 2018). However, the extent to which repeated SARA bouts affect the indicators of ruminal dysbiosis such as the release and accumulation of BA and LPS and whether PHY or AY supplementation is also able to modulate these indicators have not yet been evaluated.

Research has also established that during SARA and the resulting dysbiosis, the permeability of the rumen mucosa increases (Aschenbach and Gäbel, 2000), offering the opportunity for the microbe-derived toxic compounds to translocate into the systemic circulation. This event could lead to systemic inflammation and metabolic derailments such as displaced abomasum, laminitis, fatty liver, and plasma mineral disturbances in cattle exposed to such a SARA challenge (Zebeli and Metzler-Zebeli, 2012). Most conventional studies investigating the effect of SARA on the metabolism of cows focused on single metabolites (Zebeli et al., 2011; Marchesini et al., 2013). In recent years, however, metabolomics techniques have been developed, allowing the detection of multiple classes of metabolites that reflect changes of key metabolic pathways and help to gain a deeper understanding of the mechanisms behind metabolic disorders (Hailemariam et al., 2014a,b; Humer et al., 2016). Metabolomics-based technologies, investigating the effect of high-concentrate feeding in dairy cows carried out on rumen fluid samples, revealed substantial effects of SARA on the rumen metabolome, such as an increased concentration of several potentially toxic metabolites as well as perturbations in AA (Ametaj et al., 2010; Saleem et al., 2012). Given the potential of metabolomic technologies to extend our understanding of the mechanisms behind metabolic disorders, we hypothesized that the characterization of the blood metabolomic profile of dairy cows fed either an all-forage diet or a high-concentrate diet would provide comprehensive information on how SARA as a metabolic disease affects the cow's systemic metabolism and how these changes interact with the accumulation of toxic compounds in the rumen. Therefore, besides analyzing BA and LPS in the rumen fluid, we aimed at conducting additional blood analysis during the period of severest $\mathrm{pH}$ drops (i.e., the first SARA challenge; Kröger et al., 2017) to verify the proposed hypothesis. We used a SARA feeding challenge model with nonlactating cows to be able to investigate the effects of diet-induced ruminal disturbances on the blood metabolome, while ruling out potential confounding influences of lactation (e.g., hormonal changes, nutritional demands, and mobilization) on systemic metabolism and blood metabolome.

The aims of this study were to investigate changes in the concentration of BA and LPS in the rumen fluid as well as in the blood metabolome of cows experiencing SARA and to investigate possible modulatory effects of the supplementation of feed additives. Our respective hypothesis was that PHY and AY would help in reducing the concentration of LPS and BA in cows experiencing SARA, especially when $\mathrm{pH}$ is lowest, thereby resulting in less detrimental changes in the blood metabolome.

\section{MATERIALS AND METHODS}

\section{Animals, Diets, and Experimental Design}

The experiment was part of a larger study and detailed information about cows, feeding, and experimental setup as well as results according to reticuloruminal $\mathrm{pH}$, chewing activity, and ruminal microbiome have been reported in our companion papers (Kröger et al., 2017; Neubauer et al., 2018). In brief, 8 rumencannulated (100 mm i.d.; Bar Diamond, Parma, ID), nonlactating Holstein cows (average BW: $863 \pm 65 \mathrm{~kg}$, mean $\pm \mathrm{SD}$ ) were kept in a loose-housing stable at the research dairy farm of Vetmeduni Vienna (Pottenstein, Austria). Cows were arranged to an incomplete double $4 \times 3$ Latin square design with 4 runs and 3 treatments ( $\mathrm{n}=8$ per treatment). Cows were randomly allocated to 1 of the 3 treatment groups: control $(\mathbf{C O N}$, no additive), PHY (Digestarom Dairy, BIOMIN Holding GmbH, Getzersdorf, Austria; 3 g per animal and day) or AY (Levabon Rumen E, BIOMIN Holding GmbH; $15 \mathrm{~g}$ per animal and day). The PHY consisted of a blend of spices, herbs, and essential oils with major active compounds deriving from mint, thyme, rosemary, and clove, whereas AY contained autolyzed spray-dried yeast (Saccharomyces cerevisiae). Each of the 4 runs lasted for $43 \mathrm{~d}$ and was followed by a 3-wk washout period to allow cows to recover from high-concentrate feeding and to avoid carry-over effects.

During each experimental run, cows were exposed to an intermittent high-concentrate feeding and thus intermittent SARA conditions. Initially, cows received 
an all-forage feeding during baseline ( 1 to 7 of the experiment), consisting of a hay/grass silage mix. Afterward, the concentrate amount in the diet was gradually increased $(+9.3$ percentage points per day $)$ to reach a concentrate:forage ratio of 65:35 (DM basis) for 1 (d 14-20; SARA1 phase) or 2 wk (d 30 to 43; SARA2 phase). In between SARA1 and SARA2, cows were fed the initial all-forage diet for 1 wk during recovery (d 21-27 of the experiment). The ingredients, as well as chemical composition of the feeds used, are summarized in Supplemental Table S1 (https://doi.org/10 $.3168 /$ jds.2018-14744). Diets were offered separately via individual feeding troughs and access was regulated electronically with transponder access gates (Insentec B.V., Marknesse, the Netherlands), which enabled the recording of the daily feed intake for each individual cow.

All procedures involving animal handling and treatment were approved by the institutional ethics committee of the University of Veterinary Medicine (Vetmeduni) Vienna and the national authority according to $\S 26$ of the Law for Animal Experiments, Tierversuchsgesetz 2012- TVG (GZ: 68.205/0023-WF/V/3b/2015).

\section{Sampling Procedures}

Rumen Liquid Collection. During each experimental run, sampling was performed on d 5 (baseline), 19 (SARA1), and 39 (SARA2) $8 \mathrm{~h}$ after morning feeding. To collect particle-associated rumen liquid for determination of BA, approximately $500 \mathrm{~g}$ of digesta was taken from the middle of the rumen mat (Zebeli et al., 2008). The digesta was manually squeezed until $250 \mathrm{~mL}$ of rumen liquid were obtained. The rumen liquid was filtered through 4 layers of cheesecloth and subsamples were stored at $-20^{\circ} \mathrm{C}$ until analysis of BA. In addition, samples of rumen liquid were taken from the middle of the rumen mat using a sterile syringe to determine ruminal LPS content. The rumen liquid was directly filled in pyrogen-free 2-mL tubes (SafeSeal micro tubes, Sarstedt, Nümbrecht, Germany) and stored at $-20^{\circ} \mathrm{C}$ before being analyzed for LPS.

Analyses of Biogenic Amines. Biogenic amines (ethanolamine, methylamine, isopropylamine, pyrrolidine, putrescine, cadaverine, histamine, tyramine, spermidine, and spermine) in the rumen fluid were determined by reversed-phase HPLC (Waters 2695 Separations Module, Waters, Milford, MA) by the methods of Saarinen (2002). Separation was performed on a InertClone $5 \mu \mathrm{m}$ ODS(2) 150 A LC column 250 $\times 4.6 \mathrm{~mm}$ (Phenomenex, Torrance, CA). A UV/visible detector was used for detection (Waters 2489, Waters).

Analysis of LPS. The LPS concentration in rumen liquid was determined using the chromogenic limulus amoebocyte lysate (LAL) assay (Charles River, Reading, UK). The assay was performed using a 96-well microplate (EndoSafe, Charles River, Wilmington, MA) with absorbance read at $450 \mathrm{~nm}$ using a microplate reader (TecanSunrise, Reading, UK). Samples were thawed, transferred into glass tubes, and centrifuged at $4,500 \times g$ for $15 \mathrm{~min}$ at $4^{\circ} \mathrm{C}$ (Eppendorf 5840R, Vienna, Austria). Afterward, the supernatant was heatinactivated at $100^{\circ} \mathrm{C}$ for 15 min in a water bath (Lauda Aqualine AL 12, Beun de Ronde, Abcoude, the Netherlands). Subsequently, samples were filtered through disposable $0.45 \mu \mathrm{m}$, pyrogen-free syringe filters (Filtopour S, Sarstedt, Nürmbrecht, Germany). Filtered samples were diluted 50,000-fold using pyrogen-free LAL reagent water (Lonza, Basel, Switzerland) and 50\% glucan inhibiting buffer (Glucashield (1-3)- $\beta$-D-Glucan inhibiting buffer, catalog no. GB051-25, Cape Cod, PyroQuant Diagnostic GmbH, Mörfelden-Walldorf, Germany) was used to reconstitute LAL.

Blood Sampling and Analysis. Blood samples were collected on d 6 (baseline) and d 18 (SARA1) before morning feeding. The samples were taken from the jugular vein using 9-mL evacuated tubes (Vacuette, Greiner Bio-One GmbH, Kremsmünster, Austria) to determine the liver enzymes aspartate aminotransferase (AST), glutamate dehydrogenase (GLDH), and gamma-glutamyltransferase (GGT) and metabolites such as nonesterified fatty acids (NEFA), BHB, and cholesterol in serum. Lactate and glucose samples were collected in 6-mL fluoride plasma tubes (Vacuette, Greiner Bio-One $\mathrm{GmbH}$ ) to avoid glycolysis during storage. After sampling the serum samples were allowed to clot at room temperature for approximately $2 \mathrm{~h}$, while plasma samples were stored at $7^{\circ} \mathrm{C}$ before being separated by centrifuging at 2,000 $\times g$ for 10 min at room temperature. Subsequently, samples were stored in 2-mL tubes (Sarstedt, AG, Wiener Neudorf, Austria) at $-20^{\circ} \mathrm{C}$, until further analyses at the laboratory of the Central Clinical Pathology Unit, University of Veterinary Medicine, Vienna, using standard enzymatic colorimetric analyses. Analyses were conducted with a fully automated analyzer for clinical chemistry (Cobas 6000/c501, Roche Diagnostics GmbH, Vienna, Austria). The intraassay variation was controlled by limiting the coefficient of variation to $<5 \%$ for all blood variables.

Metabolome profiling was carried out in plasma samples using a targeted metabolomics approach as described previously (Humer et al., 2016). In brief, the Absolute-IDQ p180 platform (Kit p180, Biocrates Life Sciences AG, Innsbruck, Austria) was applied using electrospray ionization-liquid chromatography-MS/ MS. The fully automated assay allows a simultaneous quantification of 176 metabolites out of $10 \mu \mathrm{L}$ of blood 
plasma and is based on derivatization with phenylisothiocyanate. For acylcarnitines, lipids, and hexose, flow-injection analysis-MS/MS was applied, whereas liquid chromatography/MS served for the analysis of $\mathrm{AA}$ and BA. Identification of targeted classes of metabolites was performed using a AB Sciex 4000 QTrap mass spectrometer (Applied Biosystems/MDS Sciex, Foster City, CA) with electrospray ionization. The assays were performed on a double-filter 96-well plate kit containing stable isotope-labeled internal standards as a reference for the quantification of the respective metabolites. The Biocrates software (Biocrates Life Science AG) was used for analysis of mass chromatograms. Detailed information regarding the applied technique, including standards, calculations as well as the accuracy of measurements is given in Ramsay et al. (2007).

\section{Statistical Analyses}

Univariate statistical analyses were performed using the MIXED procedure of SAS (version 9.2, SAS Institute Inc., Cary, NC). All statistical models were performed with the feeding phase (baseline, SARA1, SARA2) and treatment group (CON, PHY, AY) considered as fixed effects. Interactions between feeding phase and feeding group (phase $\times$ treatment group) were also tested. For all blood variables, the same model with 2 feeding phases (baseline, SARA1) was applied. To account for repeated measurements of the same animal within a feeding group over time, a firstorder autoregressive variance-covariance structure was used according to Bayesian information criterion. Cows nested within the square and the experimental runs were considered as random effects. Treatment effects are only given for feeding phases SARA1 and SARA2 because feed additives were not administered during the baseline. Comparisons among the least squares means were performed with the pdiff option and considered as significant with $P \leq 0.05$ and as a trend with $0.05<$ $P \leq 0.10$.

For multivariate analysis, data were imported into the MetaboAnalyst 4.0 software (http://www .metaboanalyst.ca; Xia and Wishart, 2016). Data were analyzed by multivariate statistical analysis using partial least-squares discriminant analysis (PLS-DA) using MetaboAnalyst (Xia et al., 2015). The PLSDA results were visualized using the first 3 principal components of the scores plot to identify characteristic trends or grouping among cows fed the all-forage diets versus cows fed the high-concentrate diets and pertaining to group CON, PHY, or AY. Based on the PLS-DA results, the obtained variables were plotted according to their importance in separating the dietary treatments based on the variable importance in the pro- jection (VIP) scores. Hierarchical clustering analysis (HCA) with clustered heat maps was also performed with MetaboAnalyst (Xia et al., 2015) to explore the presence of clustering patterns among the rumen fluid and blood metabolites as well as ruminal $\mathrm{pH}$, forage, and grain intake during baseline and SARA1. Overall correlation pattern analysis between rumen fluid and blood metabolites as well as ruminal $\mathrm{pH}$, forage, and grain intake were conducted using the same tool.

\section{RESULTS}

\section{Effects of Intermittent SARA Challenge}

Concentration of LPS and Biogenic Amines. The effect of the intermittent SARA challenge on ruminal LPS and BA is shown in Table 1. High-concentrate feeding during SARA1 and SARA2 was associated with an 8-fold and 11-fold, respectively, increase in ruminal LPS compared with the all-forage feeding in the baseline $(P<0.01)$. With the exception of spermine, all investigated BA were increased by the elevated concentrate level in the diet. Overall, we observed a 1.6-fold increase in the concentration of BA in SARA1. Histamine showed the strongest increase with a 5.3fold higher concentration in SARA1 compared with the baseline-feeding phase $(P<0.01)$. Ethanolamine and pyrrolidine also showed highest levels in SARA1 $(P$ $<0.05)$. Isopropylamine, putrescine, cadaverine, tyramine, and spermidine were elevated during both SARA phases compared with baseline $(P<0.05)$, but did not differ between SARA1 and SARA2.

Plasma Metabolic Profile. Supplemental Table S2 (https://doi.org/10.3168/jds.2018-14744) provides a list of the blood metabolites that were affected by the concentrate-challenge (SARA1). The high-concentrate feeding increased the liver enzymes AST, GGT, and GLDH by 20,8 , and $80 \%(P<0.05)$. Cholesterol was decreased by $43 \%$ in SARA1 as compared with baseline $(P<0.01)$, whereas glucose increased by $9 \%(P<$ $0.01)$. The concentrations of lactate and NEFA were not affected by switching the cows from 0 to $65 \%$ concentrate feeding and averaged 0.70 and $0.08 \mathrm{mmol} / \mathrm{L}$, respectively.

The SARA challenge caused a shift in the plasma metabolome, such as a decrease in all detected lysophosphatidylcholines (lysoPC; $P \leq 0.01$ ) as well as a decrease of most phosphatidylcholines (PC; $P \leq$ $0.05)$ and sphingomyelins (SM; $P<0.01)$. Among the AA, glycine and serine increased, whereas most other AA decreased during SARA $(P \leq 0.07)$. Carnosine and taurine increased $(P<0.01)$, whereas other BA in plasma were either below the detection limit (i.e., acetylornithine, cis-4-hydroxyproline, dihydroxyphe- 
nylalanine, dopamine, phenylethylamine, histamine, putrescine, spermidine, spermine) or decreased in cows experiencing SARA (methionine-sulfoxide, asymmetric dimethylarginine, trans-4-hydroxyproline; $P \leq 0.02$ ). Similarly, several acylcarnitines were below the detection limit. An increase was found for malonylcarnitine (C4-OH) and oleylcarnitine (C18:1) during the SARA period $(P \leq 0.01)$, but the opposite was observed for myristoleylcarnitine (C14:1), palmitoylcarnitine (C16), and stearylcarnitine $(\mathrm{C} 18 ; P \leq 0.04)$.

To visualize the differences in the data among feeding phases, PLS-DA was conducted which showed coverage of $97.7 \%$ of the observed variation in the sample set by the first 3 principal components (Figure 1A). Cows receiving the all-forage diet clustered closer together, compared with the cows undergoing the SARA challenge, and showed a clear separation from the grouping of cows experiencing SARA. To define the most influential variables in the PLS-DA model, feature selection based on VIP scores was conducted (Figure 1B). The procedure allowed the identification of the variables that were most important for the separation among the 2 different feeding phases in the scores plot. Accordingly, the sum of hexoses, glycine, and cholesterol were the top 3 metabolites that contributed to the PLS-DA model.

\section{Effect of the Supplementation of Feed Additives}

Concentration of LPS and Biogenic Amines. Overall, most significant treatment effects were observed in SARA1. Ruminal LPS was $43 \%$ lower in cows receiving $\mathrm{PHY}$ than in CON cows in SARA1 $(P=$ 0.04; Table 2). Supplementation of the diet with PHY decreased the concentration of pyrrolidine $(-38 \%)$ and histamine $(-66 \%)$ in SARA1 (CON vs. PHY; $P=$
0.01). Moreover, PHY tended to lower the concentration of spermine in SARA1 $(-54 \% ; P=0.07)$ and of cadaverine in SARA2 $(-50 \%)$ compared with CON $(P$ $=0.08)$. For AY a decrease in ethanolamine $(-21 \%)$, methylamine $(-52 \%)$, pyrrolidine $(-35 \%)$, histamine $(-54 \%)$, spermidine $(-44 \%)$, and spermine $(-80 \%)$ compared with the CON cows was observed in SARA1 $(P<0.05)$.

Plasma Metabolic Profile. Data on the effects of feed additives on the blood metabolic profile during SARA1 are summarized in Table 3 and Figure 2. The PHY-supplemented cows had the highest plasma cholesterol concentration, differing by a trend from $\mathrm{CON}$ cows $(+26 \% ; P=0.09)$. Glucose, NEFA, BHB, lactate, $\mathrm{AST}$, and GLDH concentrations did not differ among treatment groups.

As demonstrated in Figure 2A, supplementation of PHY resulted in increases in several AA up to $34 \%$ compared with CON (i.e., arginine, asparagine, isoleucine, leucine, lysine, methionine, phenylalanine, threonine, and tyrosine; $P \leq 0.09)$. The PHY increased asymmetric dimethylarginine $(P=0.05)$ and tended to decrease creatinine by $9 \%(P=0.06)$. In PHY cows acetylcarnitines were higher $(\mathrm{C} 5, \mathrm{C} 14: 1)$ or lower (C18:1) as compared with CON cows $(P \leq 0.10)$. As demonstrated in Figure 2B, PHY increased lysoPC a (where $\mathbf{a}=$ acyl) $\mathrm{C} 14: 0$ and lysoPC a C16:0 and several PC compared with CON $(P \leq 0.10)$. Additionally, PHY increased SM (OH) C22:1 and SM C16:0 and decreased SM C22:3 $(P \leq 0.10)$. When the diet was supplemented with AY a trend toward a decrease in the AA glutamine was observed $(P=0.09)$, whereas the opposite was found for tryptophan $(P=0.08)$. Trends toward decreases in sarcosine and $\alpha$-aminoadipic acid were observed compared with CON cows $(P \leq 0.08)$, whereas $\mathrm{PC}$ aa $\mathrm{C} 40: 6$ (where aa $=$ diacyl) tended to

Table 1. Concentration of ruminal LPS (endotoxin units $/ \mathrm{mL}$ ) and biogenic amines $(\mathrm{mg} / \mathrm{kg}$ ) in cows during either an all-forage feeding (baseline) or 2 intermittent SARA feeding phases

\begin{tabular}{|c|c|c|c|c|c|}
\hline \multirow[b]{2}{*}{ Variable } & \multicolumn{3}{|c|}{ Phase $^{1}$} & \multirow[b]{2}{*}{ SEM } & \multirow[b]{2}{*}{$P$-value } \\
\hline & Baseline & SARA1 & SARA2 & & \\
\hline LPS & $14,581^{\mathrm{c}}$ & $131,209^{\mathrm{b}}$ & $168,285^{\mathrm{a}}$ & 25,722 & $<0.01$ \\
\hline Ethanolamine & $3.76^{\mathrm{c}}$ & $9.82^{\mathrm{a}}$ & $6.45^{\mathrm{b}}$ & 0.597 & $<0.01$ \\
\hline Methylamine & $37.3^{\mathrm{ab}}$ & $33.6^{\mathrm{b}}$ & $46.0^{\mathrm{a}}$ & 5.67 & 0.07 \\
\hline Isopropylamine & $7.50^{\mathrm{b}}$ & $17.4^{\mathrm{a}}$ & $16.2^{\mathrm{a}}$ & 2.65 & $<0.01$ \\
\hline Pyrrolidine & $6.68^{\mathrm{c}}$ & $12.6^{\mathrm{a}}$ & $9.41^{\mathrm{b}}$ & 1.257 & $<0.01$ \\
\hline Putrescine & $14.0^{\mathrm{b}}$ & $32.2^{\mathrm{a}}$ & $30.7^{\mathrm{a}}$ & 3.65 & $<0.01$ \\
\hline Cadaverine & $15.9^{\mathrm{Z}}$ & $24.2^{\mathrm{yz}}$ & $24.3^{\mathrm{y}}$ & 5.12 & 0.13 \\
\hline Histamine & $2.06^{\mathrm{b}}$ & $13.0^{\mathrm{a}}$ & $5.19^{\mathrm{b}}$ & 3.326 & $<0.01$ \\
\hline Tyramine & $1.00^{\mathrm{z}}$ & $1.61^{\mathrm{yz}}$ & $2.14^{\mathrm{y}}$ & 0.614 & 0.18 \\
\hline Spermidine & $8.62^{\mathrm{b}}$ & $14.9^{\mathrm{a}}$ & $17.0^{\mathrm{a}}$ & 2.417 & $<0.01$ \\
\hline Spermine & 0.70 & 1.09 & 1.10 & 0.431 & 0.40 \\
\hline
\end{tabular}

${ }^{\mathrm{a}-\mathrm{c} I n d i c a t e ~ s i g n i f i c a n t ~}(P \leq 0.05)$ changes or a trend $\left({ }^{\mathrm{y}, \mathrm{z}} 0.05<P<0.10\right)$ between the feeding phases.

${ }^{1} \mathrm{SARA} 1=65 \%$ concentrate for $1 \mathrm{wk}$; SARA $2=65 \%$ concentrate for $2 \mathrm{wk}$. 


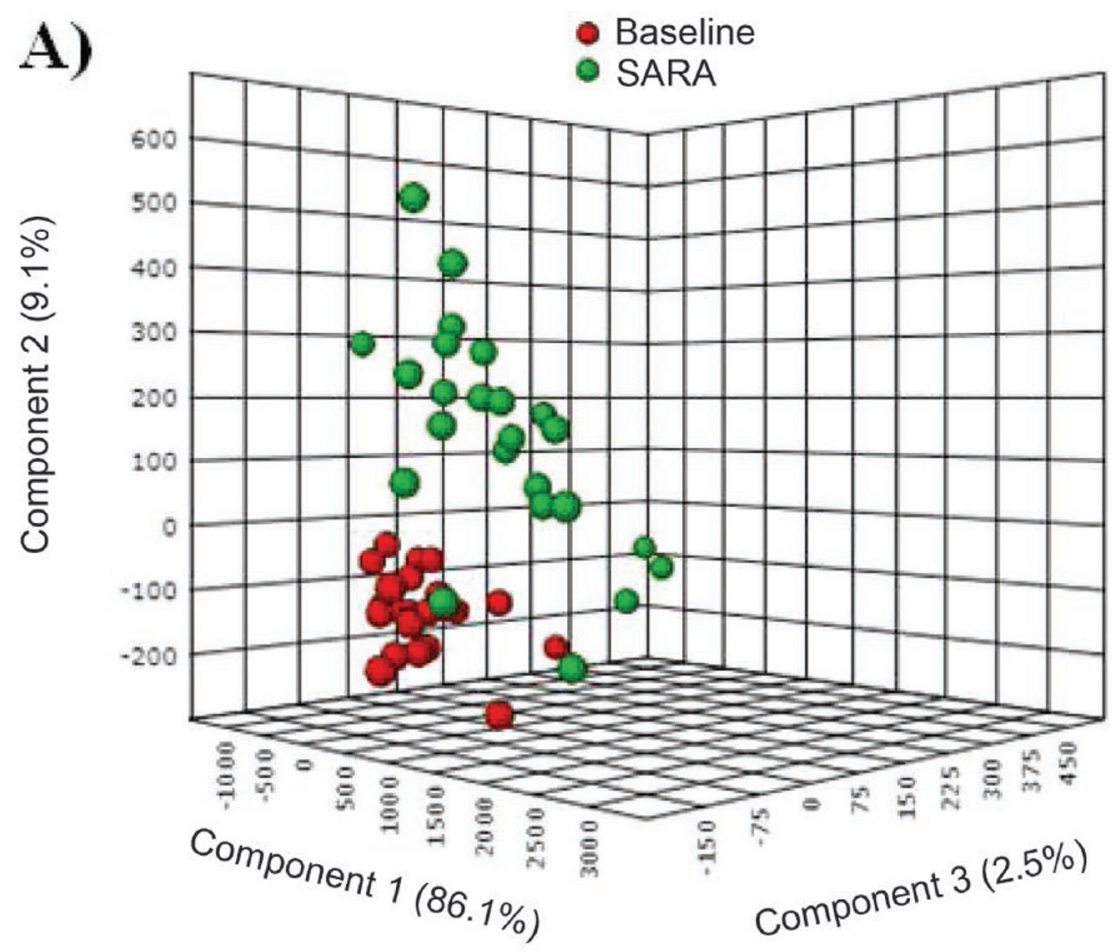

B)

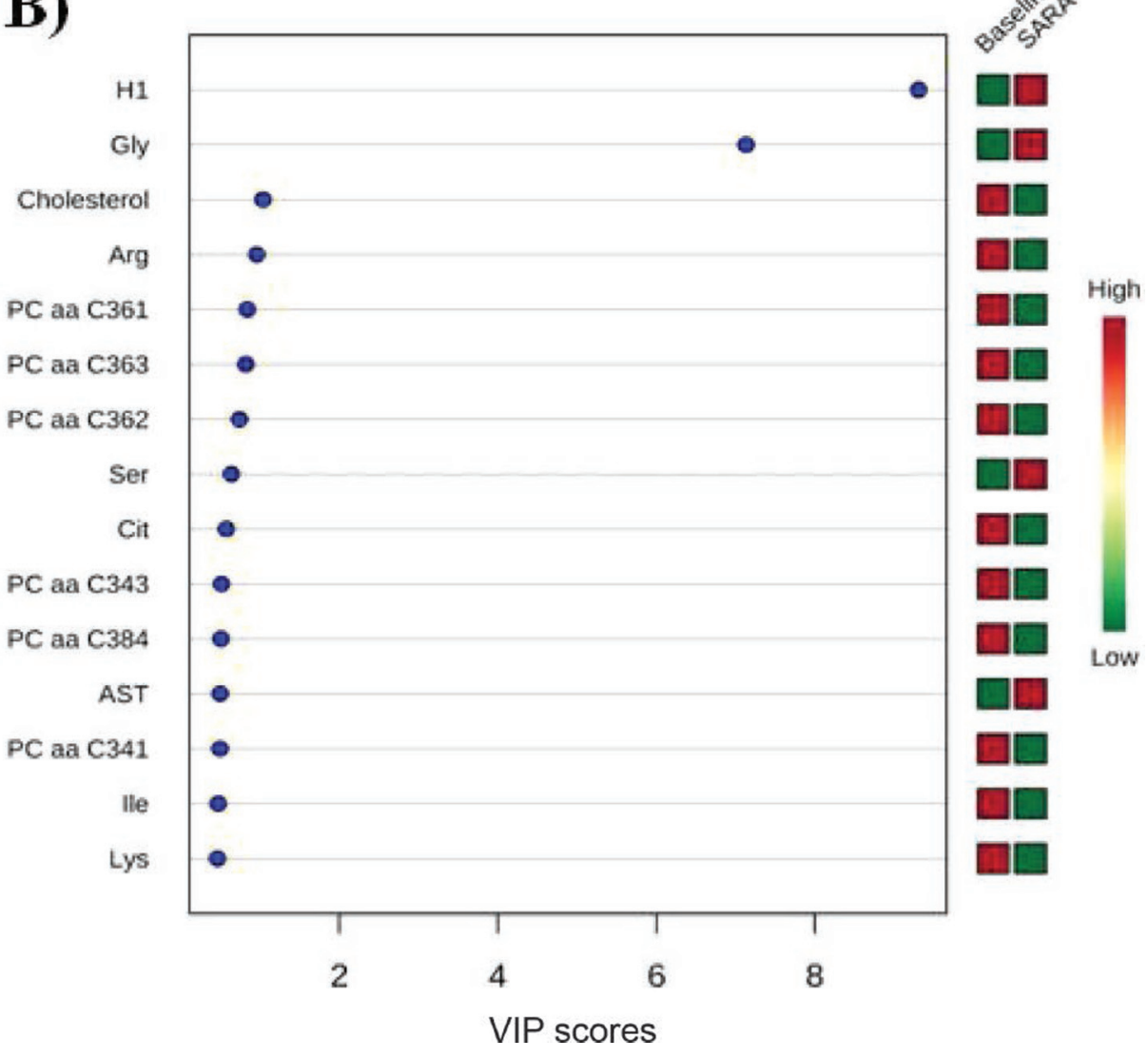

Figure 1. A partial least-squares discriminant analysis of the plasma metabolome (A). The 3-dimensional score plot distinguishes the metabolic profiles of cows fed either an all-forage diet (baseline) or a $65 \%$ concentrate diet (SARA). Variable importance in the projection (VIP) scores of the 15 most influential variables for partial least-squares discriminant analysis discriminating along principal components. $(\mathrm{B}) \mathrm{H} 1=$ the sum of hexoses; $\mathrm{PC}=$ phosphatidylcholine; aa = diacyl; AST = aspartate aminotransferase. Color version available online. 


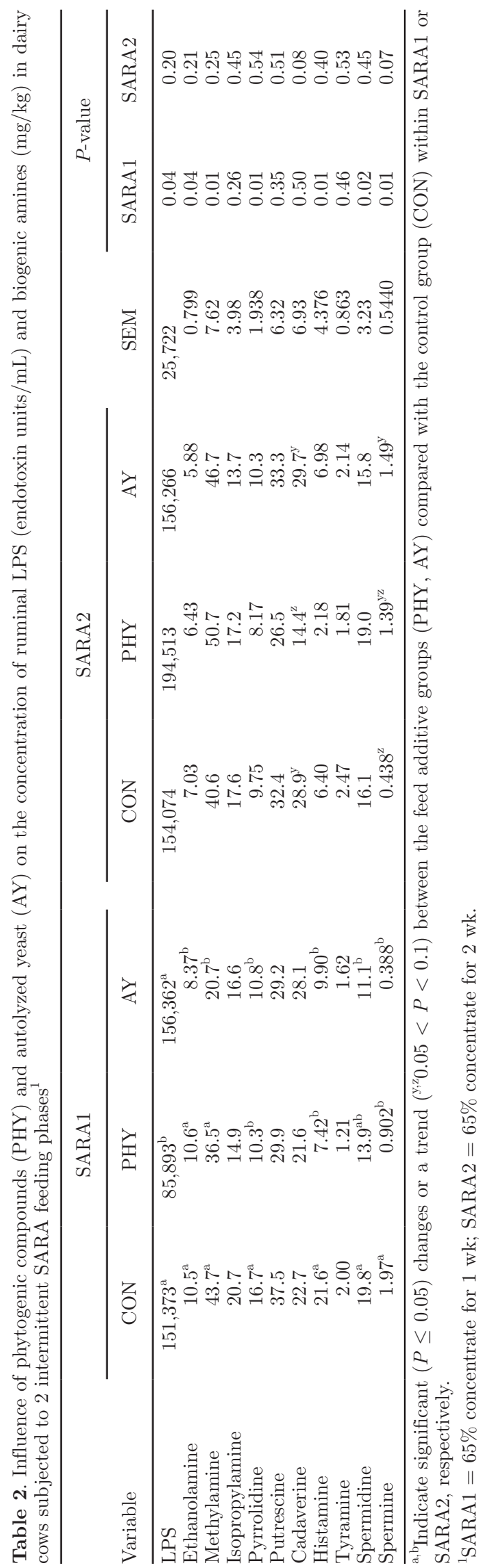

be lower in AY compared with the other groups $(P=$ $0.10)$.

To illustrate the differences in the data among feeding phases, PLS-DA was conducted that showed coverage of $97.4 \%$ of the observed variation in the sample set by the first 3 principal components, whereby the variation between metabolites can be mostly explained by component 1, whereas the contribution of component 2 and 3 was little (Figure 3A). The PLS-DA scores plot revealed that not only the baseline and SARA diets were distinct from each other, but demonstrated also that cows receiving PHY during SARA clustered closer to the baseline than CON cows. Furthermore, CON cows clustered closer together within themselves, compared with cows receiving either PHY or AY. To define the most influential variables in the PLS-DA model, feature selection based on VIP scores was conducted (Figure 3B). The procedure allowed the identification of the variables that were most important for the separation along treatment groups and baseline. Accordingly, the sum of hexoses, glycine, cholesterol, and arginine were most important for the separation of the 4 groups. Several PC and AA were also found to contribute to the separation among these groups.

\section{Relationship Between Rumen and Blood Metabolites, Ruminal pH, and Feed Intake}

To visualize the relationship and differences in the concentrations of rumen and blood metabolites, we used HCA and a heat map representation. The clustered heat map in Figure 4 shows the presence of different clusters corresponding to different numbers of metabolites with various degrees of similarity. The responses of each variable to the different diets and treatments are indicated with changes in the color intensity on the heat map. The variables with higher similarity are positioned near the top of the heat map. The HCA revealed the presence of one subcluster consisting of a group of several PC, SM (SM OH C22:2, SM C16:0), $\mathrm{C} 14: 1$, lysoPC a C16:0, cholesterol, and forage intake. An additional subcluster that is associated with the SARA diets consisted of several ruminal BA (pyrrolidine, histamine, ethanolamine), LPS, SM (SM C22:3, SM C20:2), C18:1, the grain intake, as well as the time duration the $\mathrm{pH}$ fell below the SARA threshold. Another subcluster revealed by HCA was formed by AA. A distinct feature of the HCA was that metabolites positioned in the top cluster (i.e., from PC aa C34:2 to lysoPC a C16:0) were decreased in the SARA diets, whereas the opposite was observed for the second cluster. Interestingly, the decrease in metabolites belonging to the first cluster was not or less pronounced in some cows belonging to PHY (i.e., cow number 2 and 4 ) or 
AY (i.e., cow number 2, 3, and 4). Variables clustered in the third subcluster (i.e., from phenylalanine to tryptophan) and on the bottom of the heat map showed a much more variable response.

Correlation pattern analysis was performed for all parameters that were affected by the feed additives (Figure 5). The cluster composed of PC, AA, cholesterol, and forage intake showed a negative correlation with LPS, ruminal BA, grain intake, and the time of low $\mathrm{pH}$ conditions.

\section{DISCUSSION}

\section{Effect of Intermittent SARA Challenge}

The main aim of this study was to determine changes in the concentration of LPS and BA in the rumen fluid as well as changes in the plasma metabolome during a SARA challenge in cattle. In the present study dry cows were used to exclude any lactationcycle-dependent influences on the blood metabolome. The investigated feeding model aimed at quantifying the effects of diet-induced challenge and the resulting ruminal disturbances on systemic metabolism in cows while ruling out lactational effects. We found that the change from a forage-only diet to a high-concentrate diet clearly increased the concentration of LPS and BA. Furthermore, our findings showed that the blood metabolome was considerably disrupted by the SARA challenge, involving changes in pathways of AA and lipid metabolism, as indicated by a decrease in AA, lysoPC, PC, and SM.
The strong increase of ruminal LPS, a cell-wall component of all gram-negative bacteria (GNB), during the SARA periods was expected, as high-concentrate feeding has been linked to increased death and lysis of Bacteroidetes, the majority of GNB found in the rumen, at low pH (Khafipour et al., 2009; Mao et al., 2013). Among GNB the LPS of Escherichia coli is known to be much more toxic compared with other GNB found in the cow's rumen such as Megasphera elsdenii, Prevotella, and Bacteroides (Zebeli and Metzler-Zebeli, 2012). More specifically, E. coli-derived LPS has been reported to be a major toxic compound in the rumen and to pose detrimental effects on the rumen epithelium and overall health of dairy cows (Khafipour et al., 2009; Mao et al., 2016). An interaction of BA with ruminal LPS regarding the toxicity of LPS has been suggested previously (Ametaj et al., 2010; Zebeli and Metzler-Zebeli, 2012). The present study revealed strong positive associations of LPS with ethanolamine, histamine, pyrrolidine, and spermidine as well as the duration the $\mathrm{pH}$ was below the SARA threshold. In general, the increase in BA during SARA periods is attributable to the decreased ruminal $\mathrm{pH}$. The growth of microbes that produce BA (mainly carbohydrate-fermenting bacteria such as Streptococci and Lactobacilli), as well as the activity of bacterial AA-decarboxylase, are both stimulated when ruminal $\mathrm{pH}$ decreases to an acidotic level (Straub et al., 1995; Khafipour et al., 2009; Wang et al., 2013). Although their pathophysiological functions are not yet fully understood, BA have been discussed as causative factors for decreased feed intake and reduced reticuloruminal motility (Van Os et al., 1995; Phuntsok et

Table 3. Influence of phytogenic compounds (PHY) and autolyzed yeast (AY) on the concentration of blood metabolites in cows experiencing SARA

\begin{tabular}{|c|c|c|c|c|c|}
\hline \multirow[b]{2}{*}{ Metabolite $^{1}$} & \multicolumn{3}{|c|}{ Additive } & \multirow[b]{2}{*}{ SEM } & \multirow[b]{2}{*}{$P$-value } \\
\hline & Control & PHY & $\mathrm{AY}$ & & \\
\hline Cholesterol, mg/dL & $39.3^{\mathrm{z}}$ & $49.5^{y}$ & $46.8^{\mathrm{yz}}$ & 5.69 & 0.09 \\
\hline GGT, U/L & $25.9^{\mathrm{z}}$ & $26.8^{\mathrm{yz}}$ & $28.8^{\mathrm{y}}$ & 1.73 & 0.09 \\
\hline \multicolumn{6}{|l|}{ Biogenic amine, $\mu M$} \\
\hline Asymmetric dimethylarginine & $0.662^{\mathrm{b}}$ & $0.804^{\mathrm{a}}$ & $0.755^{\mathrm{ab}}$ & 0.0560 & 0.05 \\
\hline$\alpha$-Aminoadipic acid & $1.86^{\mathrm{y}}$ & $1.84^{\mathrm{y}}$ & $1.54^{\mathrm{z}}$ & 0.182 & 0.08 \\
\hline Sarcosine & $0.900^{\mathrm{y}}$ & $1.07^{\mathrm{y}}$ & $0.530^{2}$ & 0.1489 & 0.07 \\
\hline Creatinine & $69.9^{\mathrm{y}}$ & $63.9^{\mathrm{z}}$ & $66.6^{\mathrm{y}}$ & 3.08 & 0.06 \\
\hline \multicolumn{6}{|l|}{ Acylcarnitine, $\mu M$} \\
\hline C5 & $0.069^{\mathrm{z}}$ & $0.085^{\mathrm{y}}$ & $0.084^{\mathrm{y}}$ & 0.0062 & 0.07 \\
\hline $\mathrm{C} 14: 1$ & $0.018^{\mathrm{z}}$ & $0.022^{y}$ & $0.021^{\mathrm{yz}}$ & 0.0021 & 0.10 \\
\hline C18:1 & $0.061^{\mathrm{a}}$ & $0.041^{\mathrm{b}}$ & $0.069^{\mathrm{a}}$ & 0.0074 & 0.05 \\
\hline \multicolumn{6}{|l|}{$\mathrm{SM}, \mu M$} \\
\hline $\mathrm{SM}(\mathrm{OH}) \mathrm{C} 22: 1$ & $5.78^{\mathrm{z}}$ & $6.69^{\mathrm{y}}$ & $5.92^{\mathrm{yz}}$ & 0.558 & 0.10 \\
\hline SM C16:0 & $33.1^{\mathrm{z}}$ & $38.2^{\mathrm{y}}$ & $34.6^{\mathrm{yz}}$ & 2.88 & 0.08 \\
\hline SM C20:2 & $0.034^{\mathrm{ab}}$ & $0.023^{\mathrm{b}}$ & $0.043^{\mathrm{a}}$ & 0.0081 & 0.03 \\
\hline SM C22:3 & $0.015^{\mathrm{y}}$ & $0.0040^{\mathrm{z}}$ & $0.019^{\mathrm{y}}$ & 0.00434 & 0.06 \\
\hline
\end{tabular}

${ }^{\mathrm{a}, \mathrm{b}}$ Indicate significant $(P \leq 0.05)$ changes or a trend $\left({ }^{\mathrm{y}, \mathrm{z}} 0.05<P<0.1\right)$ between the feed additive groups (PHY, AY) compared with the control group.

${ }^{1} \mathrm{GGT}=$ gamma-glutamyltransferase; $\mathrm{SM}=$ sphingomyelin. 

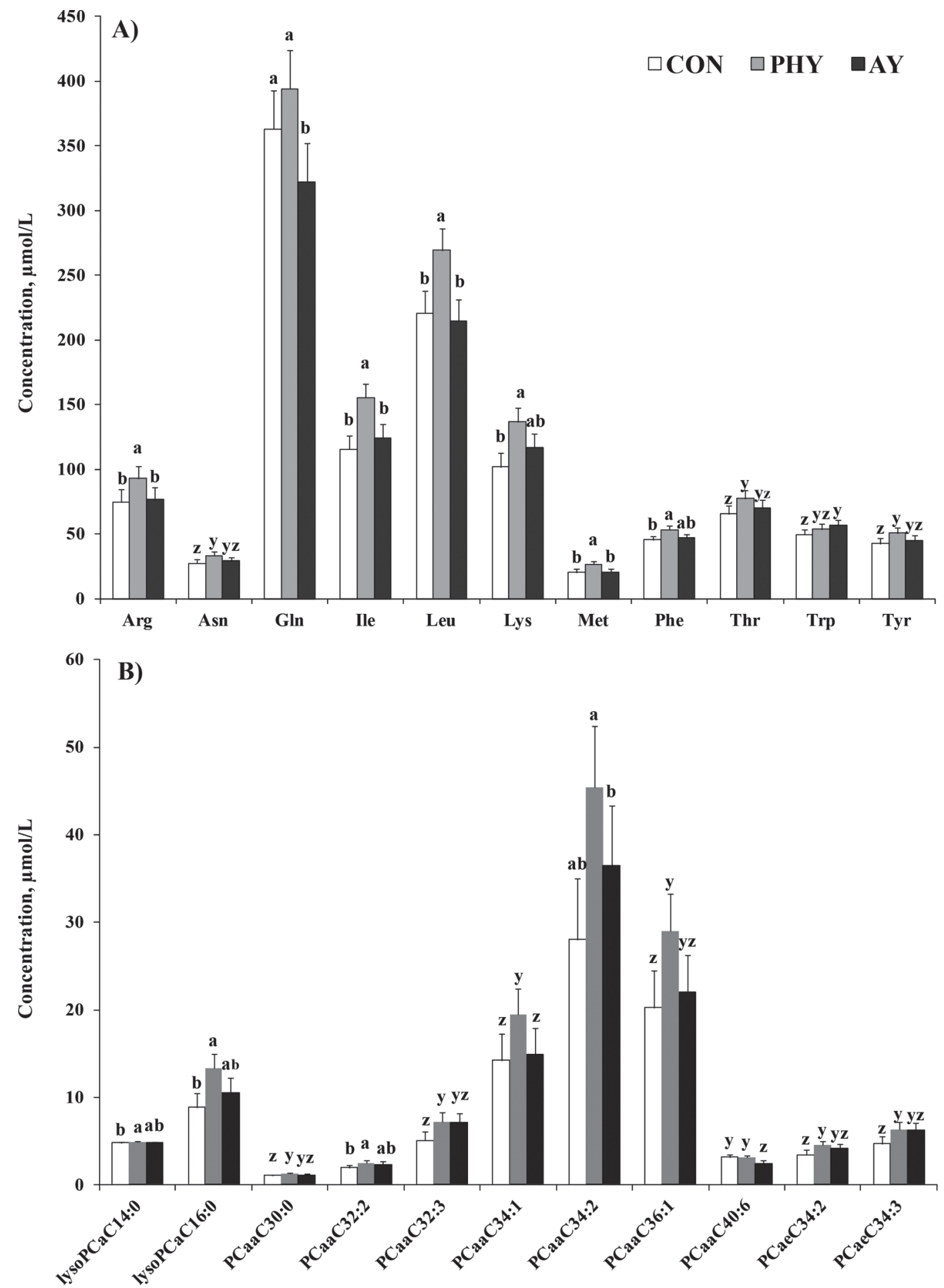

Figure 2. Concentration of AA (A), lysophosphatidylcholines and phosphatidylcholines (B) in the blood of dairy cows fed $65 \%$ concentrates and receiving no additive $(\mathrm{CON})$, phytogenic compounds $(\mathrm{PHY})$, or autolyzed yeast $(\mathrm{AY})$. lysoPC $=$ lysophosphatidylcholine; $\mathrm{PC}=$ phosphatidylcholine; $\mathrm{a}=\mathrm{acyl} ; \mathrm{aa}=$ diacyl; $\mathrm{ae}=$ acyl-alkyl. Different letters indicate significant $(\mathrm{a}, \mathrm{b} ; P \leq 0.05)$ changes or a trend $(\mathrm{y}, \mathrm{z} ; 0.05<P<0.1)$ between the feed additives. Error bars indicate SEM.

al., 1998), as well as for their detrimental effects to the rumen epithelium, such as the induction of local inflammations and impairment of the barrier function
(Zebeli and Metzler-Zebeli, 2012). Histamine is considered as one of the major toxic factors due to its putative role in laminitis (Bergsten, 2003) and the patho- 
HUMER ET AL.

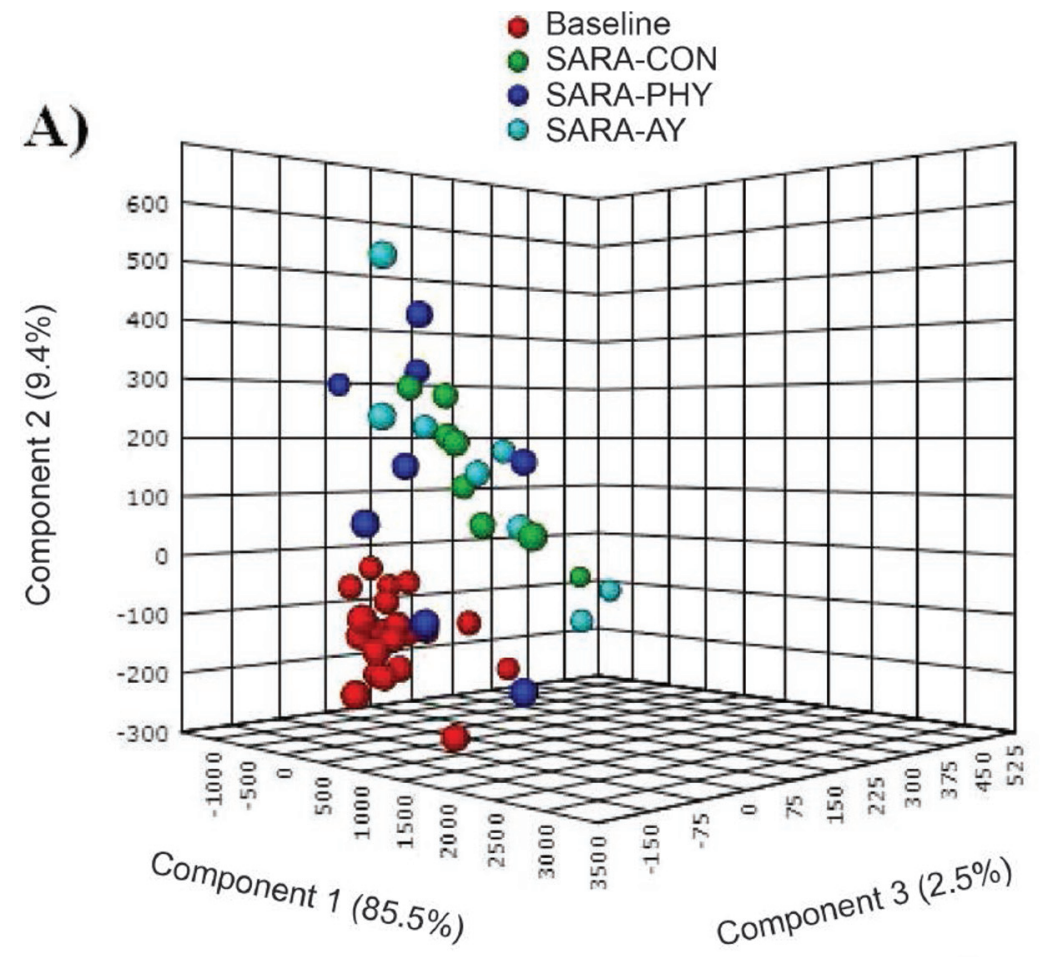

B)

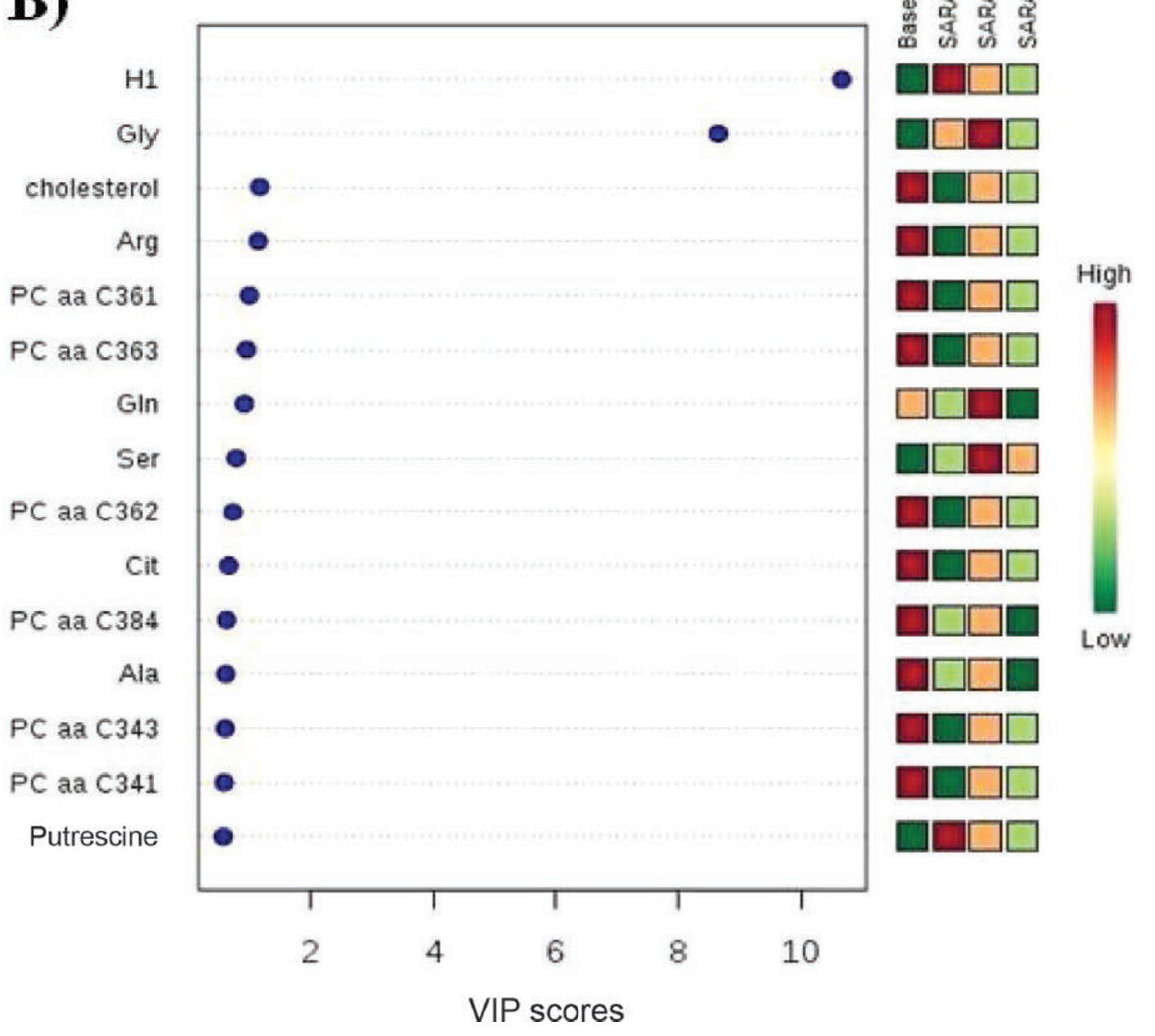

Figure 3. A partial least-squares discriminant analysis of the plasma metabolome (A). The 3-dimensional score plot distinguishes the metabolic profiles of cows fed either an all-forage diet (baseline) or a $65 \%$ concentrate diet (SARA) without feed additive (CON), or with phytogenic compounds (PHY) or autolyzed yeast (AY). Variable importance in the projection (VIP) scores of 15 most influential variables for partial leastsquares discriminant analysis (PLS-DA) discriminating along principal components (B). H1 = the sum of hexoses; PC = phosphatidylcholine; aa $=$ diacyl. Color version available online. 


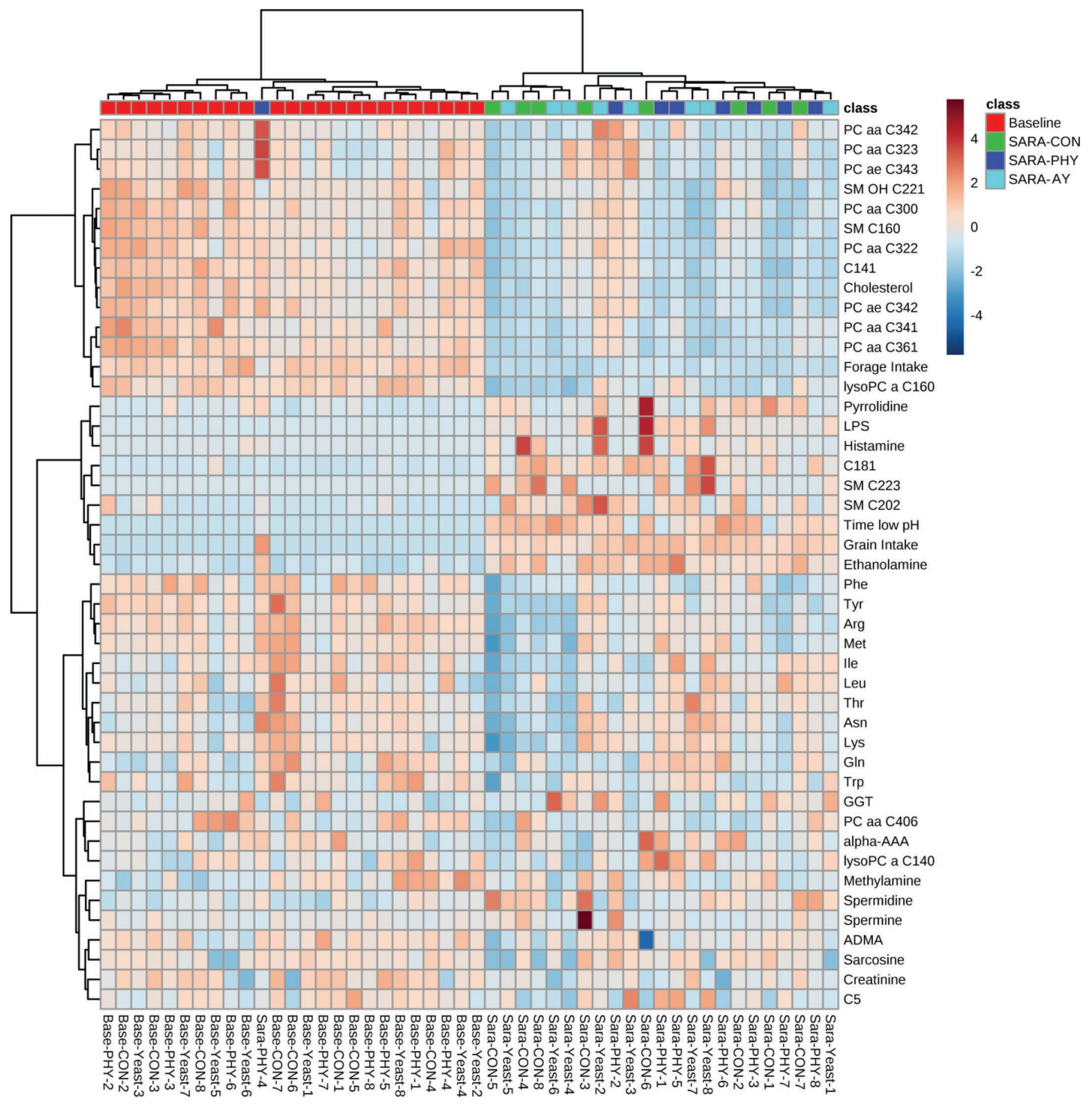

Figure 4. Hierarchical clustering analysis for different rumen fluid and blood metabolites, as well as reticular pH below the SARA threshold and forage and grain intake, measured in dairy cows receiving an all-forage diet (baseline) or $65 \%$ concentrates (SARA) and receiving no additive $(\mathrm{CON})$, phytogenic compounds (PHY), or autolyzed yeast (AY) during the SARA feeding regimen. Cells are colored based on the concentration measured in the samples. Dark red represents high levels, blue shows low levels, and gray cells show the intermediate level. The similarity between 2 variables is represented by branch height. $\mathrm{a}=$ acyl; ADMA $=$ asymmetric dimethylarginine; $\alpha$-AAA $=\alpha$-aminoadipic acid; $\mathrm{C}=$ carnitine; GGT = gamma-glutamyltransferase; $\mathrm{OH}=$ hydroxy; $\mathrm{PC}=$ phosphatidylcholine; ae = acyl-alkyl; aa $=$ diacyl; $\mathrm{SM}=$ sphingomyelin Color version available online. 


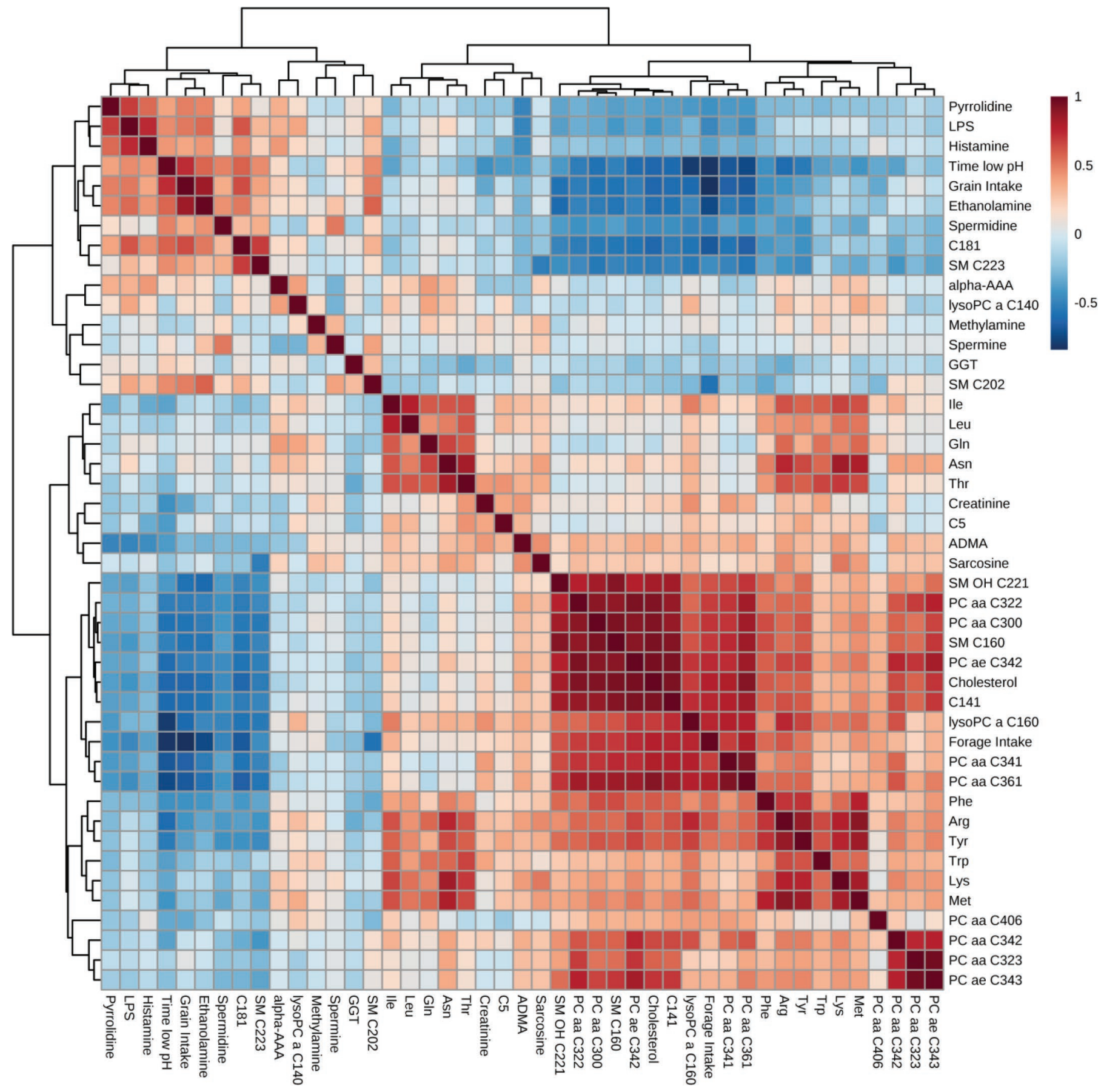

Figure 5. Correlation of the different rumen fluid and blood metabolites, as well as reticular pH below the SARA threshold and forage and grain intake measured during an all-forage feeding regimen (baseline) or a $65 \%$ concentrate-challenge (SARA) and receiving no additive (CON), phytogenic compounds (PHY), or autolyzed yeast (AY). A heat map of Spearman correlation coefficients was generated for different concentration levels. The red color indicates a positive correlation coefficient, and the blue color represents a negative coefficient. ADMA $=$ asymmetric dimethylarginine; $\mathrm{a}=$ acyl; $\alpha$-AAA $=\alpha$-aminoadipic acid; $\mathrm{C}=$ carnitine; $\mathrm{GGT}=$ gamma-glutamyltransferase; $\mathrm{OH}=$ hydroxy; $\mathrm{PC}=$ phosphatidylcholine; ae = acyl-alkyl; aa $=$ diacyl; $\mathrm{SM}=$ sphingomyelin. Color version available online.

genesis of acidosis (Aschenbach and Gäbel, 2000). In our study, SARA1 caused a more pronounced increase in the rumen concentration of histamine, but also of ethanolamine and pyrrolidine in the rumen fluid than
SARA2. This is supported by the strongest decrease in reticuloruminal pH in SARA1 (Kröger et al., 2017), as well as the enhanced abundance of Lactobacillus sp. in the respective feeding phase (Neubauer et al., 2018). 
Nevertheless, the increase in BA in the rumen liquid during SARA was not reflected in an increase in BA in the blood in the current study. A poor absorption by the rumen epithelium or rapid metabolization by methylation or oxidation in the liver when entering the systemic circulation, might be the reason behind this findings and also explain why several BA could not be detected in peripheral blood in our study (Goth, 1974; Brent, 1976).

Although BA and bacterial endotoxins have been studied intensively, the relationship between BA and LPS from the digestive tract and possible effects on the systemic metabolism remain unclear. Our multivariate analysis revealed a negative relationship between the concentration of several deleterious compounds in the rumen fluid (i.e., LPS, histamine, ethanolamine, pyrrolidine, and spermidine) and the concentration of $\mathrm{AA}, \mathrm{PC}$, lysoPC, and $\mathrm{SM}$ in the blood, suggesting that these toxic substances in the rumen liquid influenced metabolic pathways for AA and lipid metabolism. The decreasing effect of the SARA feeding on several AA concurs with previous studies reporting about a pronounced decrease in cows receiving either an external LPS challenge (Humer et al., 2018) or experiencing one or several periparturient diseases including laminitis (Hailemariam et al., 2014a). The underlying mechanism might be an increased protein catabolism driven by the requirement for $\mathrm{AA}$ as fuels for immune cells, finally causing an enhanced consumption of several AA, such as arginine or tryptophan (Le Floc'h et al., 2004; Hailemariam et al., 2014a). Besides decreasing AA, the SARA was associated with a pronounced decrease of PC, lysoPC, and SM. Decreasing ruminal PC has been reported previously (Saleem et al., 2012) and can be explained by decreasing counts of protozoa during low $\mathrm{pH}$, which are the main source of $\mathrm{PC}$ in the rumen fluid (Jouany et al., 1988; Goad et al., 1998; Khafipour et al., 2009). Decreasing PC may have resulted in the decrease of lysoPC because lysoPC are produced by hydrolysis of PC by phospholipase A2 (Hailemariam et al., 2014b). Moreover, a decrease in plasma lysoPC has been previously reported in cows receiving LPS from $E$. coli (O26:B6) intramammarily or experiencing diseases (Hailemariam et al., 2014b; Humer et al., 2018). However, as PC are generally associated with cholesterol to surround triacylglycerols (Gruffat et al., 1996), their decrease might be a common observation in cows receiving high-concentrate diets, as feeding high levels of fermentable carbohydrates is generally associated with a decreased production of acetate (Neubauer et al., 2018), the main precursor of cholesterol synthesis in ruminants (Liepa et al., 1978). Moreover, SM are part of bovine phospholipids of very low-density lipoproteins (Nilsson and Duan, 2006). This is supported by the strong positive association of PC, SM, and cholesterol, which were gathered together in one subcluster in the HCA and correlation analysis.

\section{Effect of the Supplementation of Feed Additives}

The second aim of our study was to determine the effects of the supplementation of feed additives on ruminal LPS and BA, as well as the blood metabolome when cows are challenged with high-concentrate diets. Overall, most of the effects of the feed additives on the ruminal LPS and BA occurred during SARA1, which was expected based on the strongest effect on $\mathrm{pH}$ and ruminal microbiome in SARA1, likely due to an adaptation to the concentrate-rich diet in the second challenge (Kröger et al., 2017; Neubauer et al., 2018).

Supplementation of the diet with PHY showed potential to counteract the increases in the concentration of LPS, BA, as well as changes in the blood metabolome that were caused by high-concentrate feeding. The effects on LPS and BA were most pronounced in SARA1, showing a decrease by $43 \%$ of LPS and $25 \%$ of total BA, which concurs with previous studies, demonstrating that $\mathrm{PHY}$ is most effective under low $\mathrm{pH}$ conditions (Cardozo et al., 2005). Besides the mitigating effects on ruminal LPS, the strong decrease in histamine is also expected to alleviate negative consequences of high-concentrate feeding, due to its putative role in the pathogenesis of acidosis and laminitis, as discussed above. Moreover, elevated concentrations of histamine have been speculated to exacerbate the severity of ruminal acidosis (Zhang et al., 2017). The enhanced concentration of cholesterol in SARA1 in cows receiving PHY concurs with the lowered concentration of LPS in the respective cows, as the inverse relationship between the concentration of rumen LPS and plasma cholesterol has been reported previously (Zebeli et al., 2011). An increased bile acid production to detoxify and remove LPS likely decreases blood cholesterol (Eckel and Ametaj, 2016), as cholesterol is the main precursor for the synthesis of bile acids (Parlesak et al., 2007). The increased concentration of cholesterol further supports the counteracting effect of $\mathrm{PHY}$ on the decreased concentration of plasma AA during SARA observed in CON cows, as low plasma cholesterol has been reported to be associated with disturbances of plasma AA (Chiarla et al., 2004). In addition, the effect of PHY to counteract the decrease in PC during SARA might be attributable to its increasing effect on the cholesterol concentration, as supported by their high positive correlation.

The PHY-induced reduction in ruminal LPS and BA and the subsequent changes in the systemic metabolism might be explained by different mechanisms. First, a 
decreasing effect on GNB was observed in cows receiving PHY (Neubauer et al., 2018), which likely contributed to lower release of toxic substances from GNB in the digestive tract as some GNB species harbor potential LPS producers. In agreement with PHY effects on ruminal LPS, higher blood cholesterol concentration might have been the result of a lower requirement for production of bile acids for clearance of LPS. Moreover, PHY increased ruminating activity of cows in SARA1 (Kröger et al., 2017), which likely increased salivary secretion, thus possibly diluting ruminal LPS and BA or increasing their passage out of the rumen. Furthermore, PHY may increase the reticuloruminal motility by stimulating chemo-sensitive epithelial receptors in the reticulorumen, abomasum, and duodenum (Mamaghani et al., 2013; Mendel et al., 2017). Therefore, it can be speculated that increased reticuloruminal motility enhanced the removal of deleterious compounds from the rumen as increased reticuloruminal motility also enhances digesta outflow of the reticulorumen. Decreasing BA in the PHY group seems also to be related to higher ruminal $\mathrm{pH}$ in the PHY-group (Kröger et al., 2017), as the synthesis of BA in the rumen is increased by low ruminal $\mathrm{pH}$, due to increased activity of bacterial AA-decarboxylase as discussed above.

Supplementing the diets with AY caused pronounced effects on the concentration of BA in SARA1, showing an absolute decrease by $31 \%$ compared with CON. Besides the aforementioned positive effect expected by the decreasing effect on histamine, the lowered concentration of ethanolamine, methylamine, spermidine, and spermine in cows receiving AY is also an important finding, due to their relevance for animal health. Furthermore, ethanolamine implies a risk for human health, food safety, and environmental issues, due to its critical role in multiplication of certain pathogens (Law, 2000; Saleem et al., 2012). It has been hypothesized that elevated ethanolamine concentrations, caused by a rumen microbiome shift during grain-rich feeding (Khafipour et al., 2009), accelerated the turnover rate of ruminal epithelial cells (Goodlad, 1981) and cell lysis of GNB (Nagaraja et al., 1978; Saleem et al., 2012). As ethanolamine provides a carbon or nitrogen source for many pathogens, high concentrations of this amine may pave the way for the proliferation and colonization of pathogenic GNB, such as Salmonella sp., Enterococcus sp., and E. coli, in the gastrointestinal tract (Saleem et al., 2012; Zhang et al., 2017). Methylamine might also pose a serious health risk to the cow, due to its degradation into toxic metabolites such as hydrogen peroxide and formaldehyde if absorbed into the blood circulation ( $\mathrm{Yu}$ et al., 2006). In addition, the decreasing effect of $\mathrm{AY}$ on spermidine and spermine in SARA1 is expected to counteract negative conse- quences of high-concentrate feeding, as both amines are oxidized by plasma amine oxidase into aldehyde and hydrogen peroxide in ruminants (Blaschko and Bonney, 1962; Hölttä et al., 1975). It has been hypothesized that these oxidation products contribute to metabolic diseases such as mastitis, ketosis, and fatty liver, due to their extreme toxicity for eukaryotic cells and their central role in oxidative stress, respectively (Ronchi et al., 2000). Besides affecting the concentration of BA in the rumen fluid, AY attenuated the decreasing effect of the high-concentrate feeding on plasma tryptophan. An increased tryptophan catabolism due to the administration of LPS or inflammation has been previously associated with suppressed immune responses (Melchior et al., 2004; Platten et al., 2005). Therefore, it is likely that AY counteracted inflammatory conditions.

The AY-induced reduction in BA might be explained by the stimulating effect of AY on chewing activity, which was reported previously (Kröger et al., 2017). Increased total chewing time may have led to increased salivary secretion and thus a dilution effect on BA in the gastrointestinal tract. This might have contributed to the decreased accumulation of deleterious compounds in the digestive tract. A further explanation might be the effects of AY on the ruminal microbiome (Neubauer et al., 2018). Certain yeast-cell wall components such as mannan-oligosaccharides, $\beta$-glucans, chitin, peptides, AA, and nucleotides can act as a high-affinity ligand for harmful bacteria and remove them from the digestive system without attachment and colonization (Nocek et al., 2011; Ganner and Schatzmayr, 2012). Indeed, AY decreased Bacteroides sp., a major GNB (Dworkin, 2006) as reported in our companion paper (Neubauer et al., 2018). The aforementioned yeast-cell wall components may also bind harmful substances and reduce their absorption from the digestive tract (Lei et al., 2013). Interestingly, BA were only reduced during SARA1 when AY increased minimum reticular $\mathrm{pH}$ (Kröger et al., 2017). This indicates that the stabilizing effect of AY on reticular $\mathrm{pH}$ seems to be critical for the beneficial effect of AY on BA.

\section{CONCLUSIONS}

High-concentrate feeding clearly increased the concentration of toxic compounds in the rumen liquid, with a marked increase of ethanolamine, histamine and pyrrolidine during the first SARA challenge, which was accompanied by undesirable changes in the plasma metabolomic pathways related to lipid and AA metabolism. Both the PHY and AY feed additive counteracted some of these changes and evolved their effects on the toxic compounds predominantly during low ruminal-pH conditions. Therefore, our findings suggest potential of 
the feed additives to alleviate negative consequences of high-concentrate feeding in cows. However, as the study was conducted in dry cows, one has to consider that the obtained results are not completely transferable to the situation in lactating cows. Therefore, further studies are required to evaluate the effect of SARA induction and of feed additives on the blood metabolome in lactating cows at various stages of lactation.

\section{ACKNOWLEDGMENTS}

This research was part of the K-project "ADDAAdvancement of Dairying in Austria" funded by the Austrian Research Promotion Agency (FFG, Vienna, Austria) under the scope of the Competence Centers for Excellent Technologies (COMET) program (grant no. 843543). Special thanks go to the staff of the teaching and research farm Kremesberg (Vetmeduni Vienna, Pottenstein, Austria) for the help during the feeding experiment, the laboratory team of the Institute for Animal Nutrition and Functional Plant Compounds (Vetmeduni, Vienna, Austria) as well as C. Emsenhuber (BIOMIN Holding GmbH, Tulln, Austria), A. Busch-Petersen (University of Natural Resources and Life Sciences, Vienna, Austria), and C. Privasnik for the laboratory work. Königshofer Futtermittel Assmannmühlen GmbH (Ebergassing, Austria) is acknowledged for providing the concentrate.

\section{REFERENCES}

Ametaj, B. N., Q. Zebeli, F. Saleem, S. M. Dunn, J. Xia, and D. S. Wishart. 2010. Metabolomics reveals unhealthy alterations in rumen metabolism with increased proportion of cereal grain in the diet of dairy cows. Metabolomics 6:583-594.

Aschenbach, J. R., and G. Gäbel. 2000. Effect and absorption of histamine in sheep rumen: Significance of acidotic epithelial damage. J. Anim. Sci. 78:464-470.

Bergsten, C. 2003. Causes, risk factors, and prevention of laminitis and related claw lesions. Acta Vet. Scand. Suppl. 98:157-166.

Blaschko, H., and R. Bonney. 1962. Spermine oxidase and benzylamine oxidase. Distribution, development and substrate specificity. Proc. R. Soc. Lond. 156:268-279.

Brent, B. E. 1976. Relationship of acidosis to other feedlot ailments. J. Anim. Sci. 43:930-935.

Cardozo, P. W., S. Calsamiglia, A. Ferret, and C. Kamel. 2005. Screening for the effects of natural plant extracts at different $\mathrm{pH}$ on in vitro rumen microbial fermentation of a high-concentrate diet for beef cattle. J. Anim. Sci. 83:2572-2579.

Chiarla, C., I. Giovannini, and J. H. Siegel. 2004. The relationship between plasma cholesterol, amino acids and acute phase proteins in sepsis. Amino Acids 27:97-100.

Dworkin, M. 2006. The Prokaryotes Vol 3: Archaea. Bacteria: Firmicutes, Actinomycetes. 3rd ed. M. Dworkin, S. Falkow, E. Rosenberg, K.-H. Schleifer, and E. Stackebrandt, ed. Springer, New York, NY.

Eckel, E. F., and B. N. Ametaj. 2016. Invited review: Role of bacterial endotoxins in the etiopathogenesis of periparturient diseases of transition dairy cows. J. Dairy Sci. 99:5967-5990.
Ganner, A., and G. Schatzmayr. 2012. Capability of yeast derivatives to adhere enteropathogenic bacteria and to modulate cells of the innate immune system. Appl. Microbiol. Biotechnol. 95:289-297.

Goad, D. W., C. L. Goad, and T. G. Nagaraja. 1998. Ruminal microbial and fermentative changes associated with experimentally induced subacute acidosis in steers. J. Anim. Sci. 76:234-241.

Goodlad, R. A. 1981. Some effects of diet on the mitotic index and the cell cycle of the ruminal epithelium of sheep. Q. J. Exp. Physiol. 66:487-499.

Goth, A. 1974. Medical Pharmacology.7th ed. C. B. Mosby Co, St. Louis, MO.

Gruffat, D., D. Durand, B. Graulet, and D. Bauchart. 1996. Regulation of VLDL synthesis and secretion in the liver. Reprod. Nutr. Dev. 36:375-389.

Hailemariam, D., R. Mandal, F. Saleem, S. M. Dunn, D. S. Wishart, and B. N. Ametaj. 2014a. Metabolomics approach reveals altered plasma amino acid and sphingolipid profiles associated with pathological state in transition dairy cows. Curr. Metabolomics 2:184-195.

Hailemariam, D., R. Mandal, F. Saleem, S. M. Dunn, D. S. Wishart, and B. N. Ametaj. 2014b. Identification of predictive biomarkers of disease state in transition dairy cows. J. Dairy Sci. 97:2680-2693.

Hölttä, E., P. Pulkkinen, K. Elfving, and J. Jänne. 1975. Oxidation of polyamines by diamine oxidase from human seminal plasma. Biochem. J. 145:373-378.

Humer, E., S. Aditya, and Q. Zebeli. 2018. Innate immunity and metabolomic responses in dairy cows challenged intramammarily with lipopolysaccharide after subacute ruminal acidosis. Animal. https://doi.org/10.1017/S1751731118000411. In press.

Humer, E., A. Khol-Parisini, B. U. Metzler-Zebeli, L. Gruber, and Q. Zebeli. 2016. Alterations of the lipid metabolome in dairy cows experiencing excessive lipolysis early postpartum. PLoS One 11. https://doi.org/10.1371/journal.pone.0158633.

Jouany, J. P., D. I. Demeyer, and J. Grain. 1988. Effect of defaunating the rumen. Anim. Feed Sci. Technol. 21:229-265.

Khafipour, E., S. Li, J. C. Plaizier, and D. O. Krause. 2009. Rumen microbiome composition determined using two nutritional models of subacute ruminal acidosis. Appl. Environ. Microbiol. 75:7115-7124.

Kröger, I., E. Humer, V. Neubauer, N. Reisinger, S. Aditya, and Q. Zebeli. 2017. Modulation of chewing behavior and reticular $\mathrm{pH}$ in nonlactating cows challenged with concentrate-rich diets supplemented with phytogenic compounds and autolyzed yeast. J. Dairy Sci. 100:9702-9714.

Law, D. 2000. Virulence factors of Escherichia coli O157 and other Shiga toxin-producing E. coli. J. Appl. Microbiol. 88:729-745.

Le Floc'h, N., D. Melchior, and C. Obled. 2004. Modifications of protein and amino acid metabolism during inflammation and immune system activation. Livest. Prod. Sci. 87:37-45.

Lei, C. L., G. Z. Dong, L. Jin, S. Zhang, and J. Zhou. 2013. Effects of dietary supplementation of montmorillonite and yeast cell wall on lipopolysaccharide adsorption, nutrient digestibility and growth performance in beef cattle. Livest. Sci. 158:57-63.

Liepa, G. U., D. C. Beitz, and J. R. Linder. 1978. Cholesterol synthesis in ruminating and nonruminating goats. J. Nutr. 108:535-543.

Mamaghani, A., M. Maham, and B. Dalir-Naghadeh. 2013. Effects of ginger extract on smooth muscle activity of sheep reticulum and rumen. Vet. Res. Forum 4:91-97.

Mao, S. Y., W. J. Huo, and W. Y. Zhu. 2016. Microbiome-metabolome analysis reveals unhealthy alterations in the composition and metabolism of ruminal microbiota with increasing dietary grain in a goat model. Environ. Microbiol. 18:525-541.

Mao, S. Y., R. Y. Zhang, D. S. Wang, and W. Y. Zhu. 2013. Impact of subacute ruminal acidosis (SARA) adaptation on rumen microbiota in dairy cattle using pyrosequencing. Anaerobe 24:12-19.

Marchesini, G., R. De Nardi, M. Giansella, A.-L. Stefani, M. Morgante, A. Barberio, I. Andrighetto, and S. Segato. 2013. Effect of induced ruminal acidosis on blood variables in heifers. BMC Vet. Res. 9:98. 
Melchior, D., B. Séve, and N. Le Floc'h. 2004. Chronic lung inflammation affects plasma amino acid concentrations in pigs. J. Anim. Sci. 82:1091-1099.

Mendel, M., J. Chlopecka, N. Dziekan, and W. Karlik. 2017. Phytogenic feed additives as potential gut contractility modifiers-A review. Anim. Feed Sci. Technol. 230:30-46.

Nagaraja, T. G., E. E. Bartley, L. R. Fina, and H. D. Anthony. 1978. Relationship of rumen gram-negative bacteria and free endotoxin to lactic acidosis in cattle. J. Anim. Sci. 47:1329-1337.

Neubauer, V., R. Petri, E. Humer, I. Kröger, E. Mann, N. Reisinger, M. Wagner, and Q. Zebeli. 2018. High-grain diets supplemented with phytogenic compounds or autolyzed yeast modulate ruminal bacterial community and fermentation in dry cows. J. Dairy Sci. 101:2335-2349.

Nilsson, Å., and R.-D. Duan. 2006. Absorption and lipoprotein transport of sphingomyelin. J. Lipid Res. 47:154-171.

Nocek, J. E., M. G. Holt, and J. Oppy. 2011. Effects of supplementation with yeast culture and enzymatically hydrolyzed yeast on performance of early lactation dairy cattle. J. Dairy Sci. 94:40464056

Parlesak, A., S. Schaeckeler, L. Moser, and C. Bode. 2007. Conjugated primary bile salts reduce permeability of endotoxin through intestinal epithelial cells and synergize with phosphatidylcholine in suppression of inflammatory cytokine production. Crit. Care Med. 35:2367-2374.

Phuntsok, T., M. A. Froetschel, H. E. Amos, M. Zeng, and Y. W. Huang. 1998. Biogenic amines in silage, apparent postruminal passage, and the relationship between biogenic amines and digestive function and intake by steers. J. Dairy Sci. 81:2193-2203.

Platten, M., P. P. Ho, S. Youssef, P. Fontoura, H. Garren, E. M. Hur, R. Gupta, L. Y. Lee, B. A. Kidd, W. H. Robinson, R. A. Sobel, M. L. Selley, and L. Steinman. 2005. Treatment of autoimmune neuroinflammation with a synthetic tryptophan metabolite. Science 310:850-855.

Plaizier, J. C., E. Khafipour, S. Li, G. N. Gozho, and D. O. Krause. 2012. Subacute ruminal acidosis (SARA), endotoxins and health consequences. Anim. Feed Sci. Technol. 172:9-21.

Ramsay, S. L., W. M. Stoegg, K. M. Weinberger, A. Graber, and W. Guggenbichler. 2007. Apparatus and method for analyzing a metabolite profile. EP 1875401 A211-Jan-2007.

Ronchi, B., U. Bernabucci, N. Lacetera, and A. Nardone. 2000. Oxidative and metabolic status of high yielding dairy cows in different nutritional conditions during the transition period. Page 125 in Proc. 51st Annual Mtg. EAAP, Vienna, Austria. Wageningen Pers., Wageningen, the Netherlands.
Saarinen, M. T. 2002. Determination of biogenic amines as dansyl derivatives in intestinal digesta and feces by reversed phase HPLC. Chromatographia 55:297-300.

Saleem, F., B. N. Ametaj, S. Bouatra, R. Mandal, Q. Zebeli, S. M. Dunn, and D. S. Wishart. 2012. A metabolomics approach to uncover the effects of grain diets on rumen health in dairy cows. J. Dairy Sci. 95:6606-6623.

Straub, B. W., M. Kicherer, S. M. Schilcher, and W. P. Hammes. 1995. The formation of biogenic amines by fermentation organisms. Eur. Food Res. Technol. 201:79-82.

Tajima, K., S. Arai, K. Ogata, T. Nagamine, H. Matsui, M. Nakamura, R. I. Aminov, and B. Yoshimi. 2000. Rumen bacterial community transition during adaptation to high-grain diet. Anaerobe 6:273-284

Van Os, M., J. P. Dulphy, and R. Baumont. 1995. The effect of protein degradation products in grass silages on feed intake and intake behaviour in sheep. Br. J. Nutr. 73:51-64.

Wang, D. S., R. Y. Zhang, W. Y. Zhu, and S. Y. Mao. 2013. Effects of subacute ruminal acidosis challenges on fermentation and biogenic amines in the rumen of dairy cows. Livest. Sci. 155:262-272.

Xia, J., I. Sinelnikov, B. Han, and D. S. Wishart. 2015. MetaboAnalyst 3.0-Making metabolomics more meaningful. Nucleic Acids Res. $43: 251-257$

Xia, J., and D. S. Wishart. 2016. Using MetaboAnalyst 3.0 for comprehensive metabolomics data analysis. Curr. Protoc. Bioinformatics 55:14.10.1-14.10.91.

Yu, P., H. Xin, L. Lu, H. Fan, M. Kazachkov, Z. J. Jiang, S. Jalkanen, and C. Stolen. 2006. Involvement of semicarbazide-sensitive amine oxidase-mediated deamination in lipopolysaccharide-induced pulmonary inflammation. Am. J. Pathol. 168:718-726.

Zebeli, Q., S. M. Dunn, and B. N. Ametaj. 2011. Perturbations of plasma metabolites correlated with the rise of rumen endotoxin in dairy cows fed diets rich in easily degradable carbohydrates. J. Dairy Sci. 94:2374-2382.

Zebeli, Q., and B. U. Metzler-Zebeli. 2012. Interplay between rumen digestive disorders and diet-induced inflammation in dairy cattle. Res. Vet. Sci. 93:1099-1108.

Zebeli, Q., M. Tafaj, B. Junck, D. Mansmann, H. Steingass, and W. Drochner. 2008. Effects of dietary forage particle size and concentrate level on fermentation profile, in vitro degradation characteristics and concentration of liquid- or solid-associated bacterial mass in the rumen of dairy cows. Arch. Anim. Nutr. 62:230-240.

Zhang, R., W. Zhu, L. Jiang, and S. Mao. 2017. Comparative metabolome analysis of ruminal changes in Holstein dairy cows fed low- or high-concentrate diets. Metabolomics 13:74. 